\title{
Demonstrating Reduced Gravity
}

\author{
Howard Pearlman, Dennis Stocker, Daniel Gotti, David Urban, \\ Howard Ross, and Thomas Sours
}

NASA Lewis Research Center, MS 500-115, 21000 Brookpark Road, Cleveland, OH 44135

T he effects of gravity are so inherent in life on Earth that we often ignore how these effects complicate and sometimes mask fundamental processes in chemical and physical systems. To highlight the importance of gravity, we built a series of experiments, appropriate for the classroom, to demonstrate how the behavior of common physical systems change when gravity is nearly eliminated.

Based on Newton's law, $F=m a$, Einstein realized that a body of weight $W=m g$ while stationary on the Earth, would appear weightless if it were in free fall. Imagine that the body is within an imaginary box (i.e., frame of reference) that is falling at the Earth's gravitational acceleration $(a=g)$. Relative to the falling frame of reference, the body is motionless, there is no net acceleration, and therefore $W=0$. The state of free fall is often referred to as weightlessness or microgravity.

In several research labs around the world, scientists take advantage of this idea and perform "gravity-free" experiments in drop towers, airplanes, and spacecraft. Drop towers typically provide several seconds of microgravity and, as the name suggests, operate by hoisting the experiments to the top of a tower and dropping them. For example, NASA Lewis has two towers; one provides a fall distance of about $25 \mathrm{~m}$ and the other provides $130 \mathrm{~m}$. These fall distances correspond to 2.2 and $5.2 \mathrm{~s}$ of microgravity time, respectively. The Japanese Space Environment Utilization Center in Hokkaido, Japan has a 10-s drop tower with a fall distance of about $500 \mathrm{~m}$, roughly one-third of a

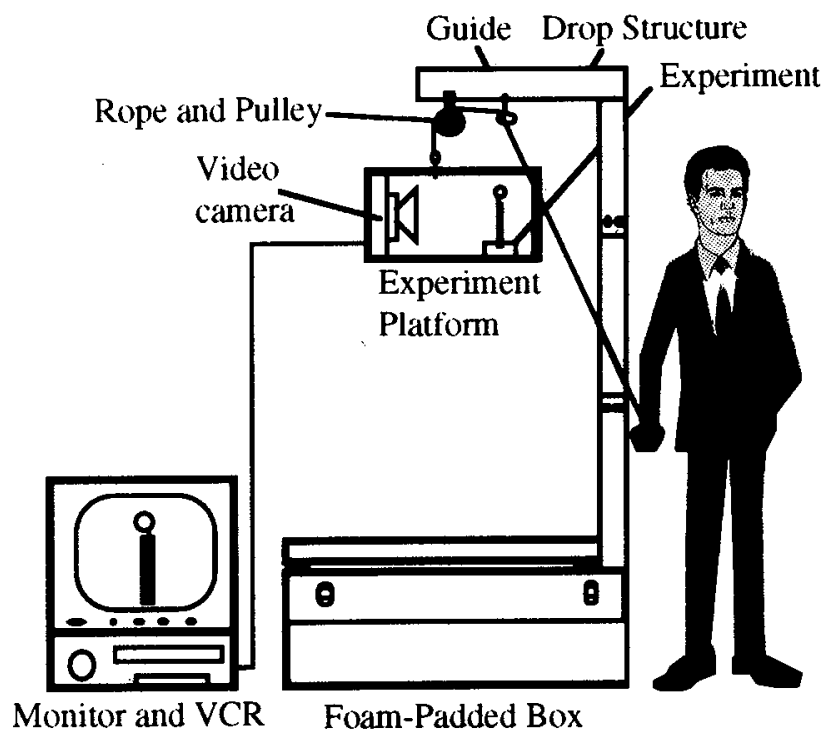

Fig. 1. Reduced-Gravity Demonstrator.

mile. Similarly, free-fall experiments are performed aboard airplanes, sounding rockets, and the Space Shuttle by flying a trajectory such that the craft falls freely towards the Earth (and around it in the case of the Space Shuttle).

Using this same principle, we developed a miniature drop tower at NASA Lewis, named the Reduced-Gravity Demonstrator (Fig. 1), to illustrate the effects of gravity on a variety of phenomena including the way fluids flow, flames burn, and mechanical systems (such as a pendulum) behave. It comes equipped with several interchangeable experiments, called "payloads." A schematic and description of the demonstrator and payloads are given here, followed by suggestions for how you can build your own. In addition, duplicate demonstrators will be distributed to NASA Centers nationwide.

\section{The Reduced-Gravity Demonstrator}

The Reduced-Gravity Demonstrator consists of four essential parts: (1) an experiment platform, (2) a drop structure to support the platform as it is hoisted to the top and released into freefall, (3) a foam-padded crate to catch the platform as it lands, and (4) a video cassette recorder (VCR) and a monitor to record the experiment during the drop and replay it afterwards.

(1) The experiment platform consists of a video camera and one of several interchangeable experiment payloads 


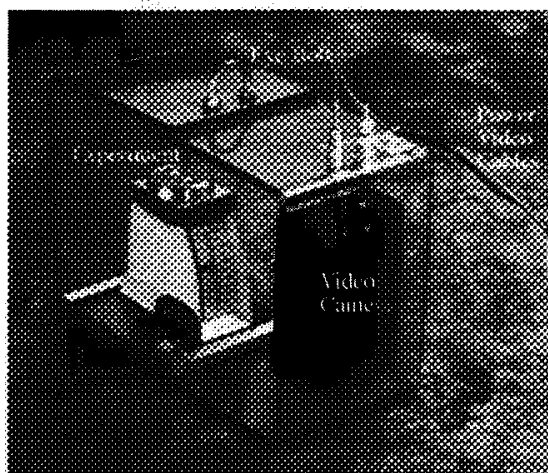

Fig. 2. Experiment platform.

(described below). The experiment payloads use base plates of a common size for simple attachment. An eye bolt is fastened to the platform so it can be hoisted to the top of the drop structure using a rope and pulley. The drop is initiated by simply releasing the rope.

(2) The drop structure provides a rigid means by which one can easily raise and release the experiment platform (see Fig. 2). The structure is segmented for easy assembly and provides a free-fall distance of approximately 2 $\mathrm{m}$. Thus, the platform and experiment will free fall for about $0.6 \mathrm{~s}$.

(3) The foam-padded crate cushions the impact when the platform hits the bottom, and as shown in Fig. 1, a mount for the drop structure is fastened to its internal side wall.

(4) The video system consists of a video recorder and monitor that are connected to the video camera mounted on the experiment platform. A long cable is connected from the "video out" port of the camera (on the platform) to the input of the VCR. The cable is approximately $4 \mathrm{~m}$ in length to allow unrestricted motion of the platform during the drop. The output of the VCR is then connected to the input of the monitor. It is helpful to use a video recorder with a frame-byframe jog shuttle (or slow motion) to replay the video slowly since the test time is short $(0.6 \mathrm{~s})$.

\section{The Payloads}

Here are several sample experiments that we use to illustrate the physics of fluids, flames, and mechanical systems. Their behavior is shown both at normal gravity $(1 g)$ and also during the drop when the experiment is in microgravity $(\mu g)$.

Weightlessness. To demonstrate the principle of weightlessness, a scale holding a mass is dropped. The two forces that counterbalance each other in this experiment are the force of the spring in the scale and the gravitational force acting on the mass, i.e., the weight. Prior to the drop, the weight of the mass balances the repulsive force of the spring such that the net acceleration of the payload is zero $(\Sigma F=m a=k x-m g$ $=0$, where $k$ is the spring constant and $x$ is the displacement of the spring as indicated by the location of the arrow on the scale). During the drop, the only force acting on the weight, relative to the payload, is the spring force $(\Sigma F=k x$ $=0 \rightarrow x=0$ ), so the indicator on the scale moves toward zero.

The only special material needed for this experiment is a small scale (dietary or postage) with a weight fastened to the top (see Fig. 3). The weight should be appropriately chosen so that the indicator reads in the middle of the scale before initiation of the drop. We also recommend putting a piece of foam between the weight and the top of the scale to cushion the impact when the package hits the bottom.

Fluid Interface. The fluid-interface experiment highlights the importance of surface tension in the absence of gravity.
In $1 g$, surface tension is evident only near the container walls while most of the surface is flat. In $\mu g$, surface tension leads the liquid to assume a very different shape since the liquid tends to creep up the container walls by capillary forces. This is most evident in the corners. Given enough $\mu g$ time, the liquid would wet all of the walls of the vessel, leaving a bubble of air in the center.

For this experiment, you will need a closed container partially filled with fluid (see Fig. 4). It is helpful if the container has sharp corners where the effect is most evident. Our acrylic container uses a flat front with a slightly curved back, giving two sharp corners. For the fluid, we recommend a low viscosity silicon oil. Water does not show the gravitational effects well because of its large (relative to silicon oil) contact angle. Other fluids may be used, but their viscosities must be low for the effects of gravity to be evident in the fall time.

Candle Flame. The candle-flame experiment demonstrates the effect of buoyant convection and its absence on combustion phenomena. In $1 g$, the combustion gases are much hotter, and thus lighter than the surrounding air. Buoyancy causes the hotter, less dense combustion gases to rise, giving the candle flame its vertically elongated, conical shape. However, during the drop, the hot

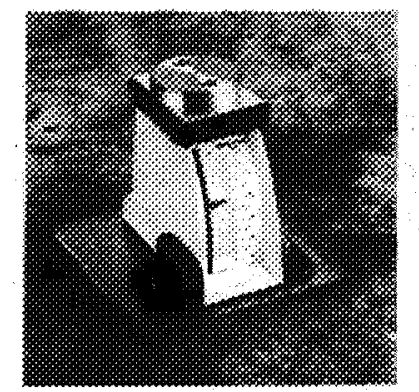

Fig. 3. Weightlessness experiment.

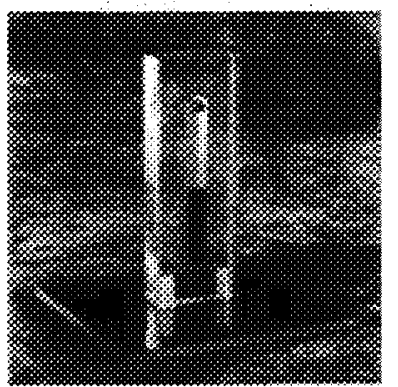

Fig. 4. Fluld-Interface experiment.

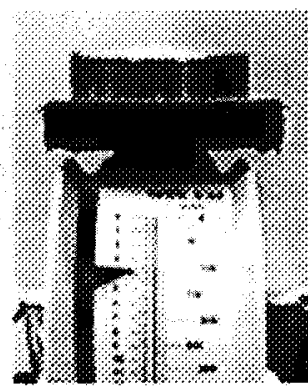

$1 g$

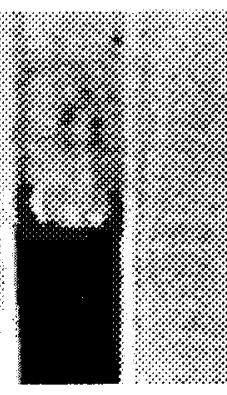

$1 g$

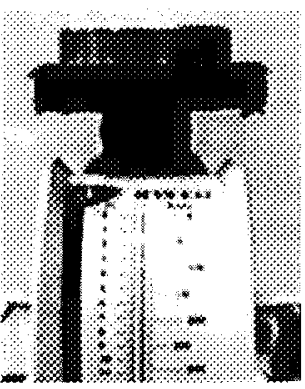

$\mu g$ 


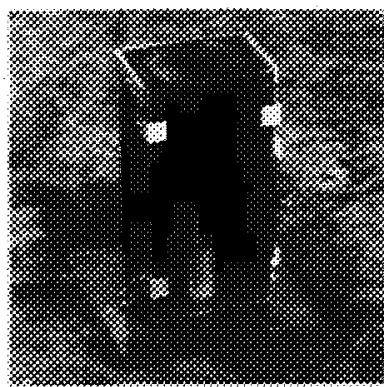

Fig. 5. Candle-flame experiment.

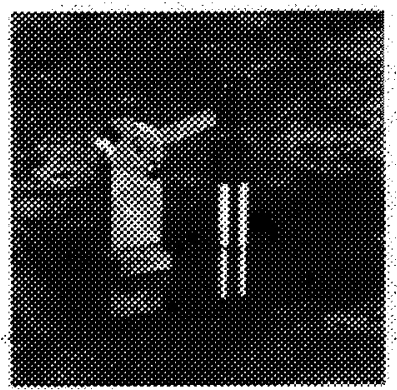

Fig. 6. Magnetic-repulsion experiment

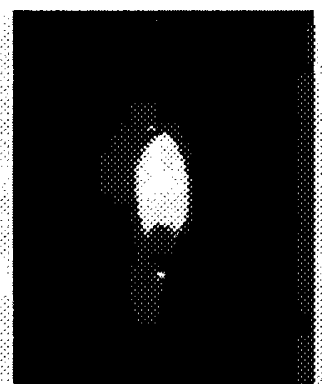

$\lg$

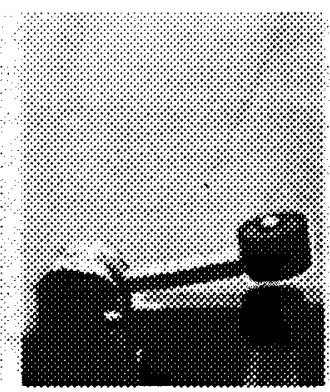

19

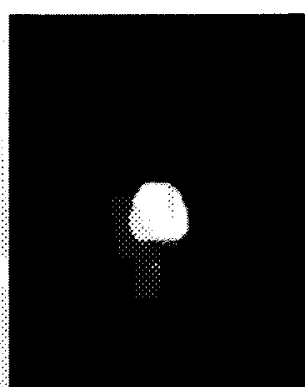

$\mu g$

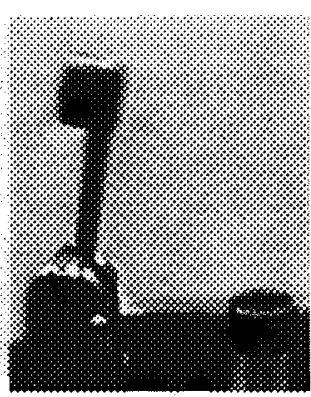

$\mu g$ gas expands in all directions since warm air no longer rises. As a result, the flame becomes shorter and wider. In longer periods of $\mu g$, the flame becomes spherical and entirely blue. This was observed in a candle flame experiment performed on the Space Shuttle (USML-1/STS-50, June-July 1992).

This experiment requires a candle with an enclosure to protect it from air drafts during the drop (see Fig. 5). Otherwise, the candle tends to flicker, obscuring observation of its microgravity behavior. The enclosure must have an opening for air exchange or the candle will quickly extinguish due to depletion of oxygen. Our best success was with a single opening at the top, covered with a fine wire mesh to limit the drafts, and a small door at the base of the enclosure to allow for ignition. This door was closed seconds before the drop; otherwise the flame would extinguish. It may be possible to adapt a small lantern from a camping supply store as the enclosure.

Magnetic Repulsion. Two magnets are oriented with like polarities opposing one another (i.e., $\mathrm{N}-\mathrm{N}$ or S-S). The lower magnet is fixed to the experiment platform while the upper magnet is freely supported on a lever arm. In $1 g$, the upper magnet is levitated by the repulsive force of the lower magnet balanced by the gravitational force pulling the upper magnet downward; i.e., $F_{\text {repulsive }}-m g=0$. During the drop, the only force acting in the falling reference frame is that due to the magnets. Hence, the separation distance between the magnets increases.

This experiment requires two magnets. One should be fastened to the pay- load base while the other should be suspended above it. We have fastened the upper magnet to a pivoting support, yet there are many different ways to do it (see Fig. 6). For instance, if you have two doughnut-shaped magnets, you could simply attach one magnet to the base, mount a vertical rod through its center hole, and then suspend the second magnet above the first, using the rod as a guide. A stop should be added at the top of the rod to prevent the magnets from falling off during the drop or upon deceleration. We discovered that ceramic magnets often break upon direct impact. We recommend avoiding them for this experiment or protecting them with an intermediate layer of foam.

We encourage you to devise and try some of your own "drop tower" experiments. These might include studying how and why air bubbles rise in a liquid (demonstrated by placing an antacid tablet in water and dropping it), how fluids flow, how a mass on the end of a rubber band moves, or how the motion of a pendulum changes when it is dropped. For further information on how to incorporate lessons on microgravity into your curriculum, we encourage you to get a copy of NASA's brochure ${ }^{1}$ describing the ReducedGravity Demonstrator along with the teachers' guide. ${ }^{2}$ These are available on the World Wide Web (WWW) at http://zeta.lerc.nasa.gov or from your nearest NASA Teacher Resource Center (see the list below). The guide briefly discusses the history of gravity and the relationship between weightlessness and free fall, then describes a number of other experiments pertaining to gravity

\section{NASA Teacher Resource Centers}

NASA Ames Research Center, Mail Stop TO-25, Moffet Field, CA 94035

NASA Goddard Space Flight Center, Mail Code 130.3, Greenbelt, MD 20771

NASA Johnson Space Center, Mail Code AP-4, Houston, TX 77058

NASA Langley Research Center, Mail Stop 146, Hampton, VA 23665

NASA Lewis Research Center, MS 8-1, 21000 Brookpark Road, Cleveland, OH 44135

NASA Kennedy Space Center, Mail Code ERL, Kennedy Space Center, FL 32899

NASA John Stennis Space Center, Building 1200, Stennis Space Center, MS 39529

Jet Propulsion Laboratory, MS CS-530, 4800 Oak Grove Drive, Pasadena, CA 91109

U.S. Space and Rocket Center, Huntsville, AL 35807
415-604-3574

$301-286-8570$

713-483-8696

804-864-3293

216-433-2017

407-867-4090

601-688-3338

818-354-6916

205-544-5812 
that can be easily demonstrated to students.

\section{Alternative Suggestions}

If you do not wish to invest the time and money into building the ReducedGravity Demonstrator discussed here, we offer some alternatives. First, you can eliminate the drop structure by simply suspending a pulley from the ceiling. Also, you can use a cushioned cardboard box for the deceleration system at the end of the drop. The experiment platform can be something as simple as a wooden board to which the camera and experiment are mounted. We do, however, suggest that you use some kind of "roll bar" or protective covering on the sides of the board to protect the camera and experiment on impact. A good choice for the video camera is a "board" camera, which is literally a circuit board with a lens mounted on it. These cameras are generally small (order of several cubic cm), cost about $\$ 200$, and can be purchased from many electric and video supply companies. Most other video cameras are also solid-state and sufficiently rugged, although we discourage the use of camcorders due to the fragile nature of their recording mechanism. Have fun and enjoy exploring the wonderful, abstract nature of gravity.

\section{References}

1. "Reduced-Gravity Demonstrator" (B0797) (NASA, 1995).

2. "Microgravity: A Teacher's Guide with Activities for Physical Science" (EG103), edited by G.L. Vogt and M.W. Wargo. 